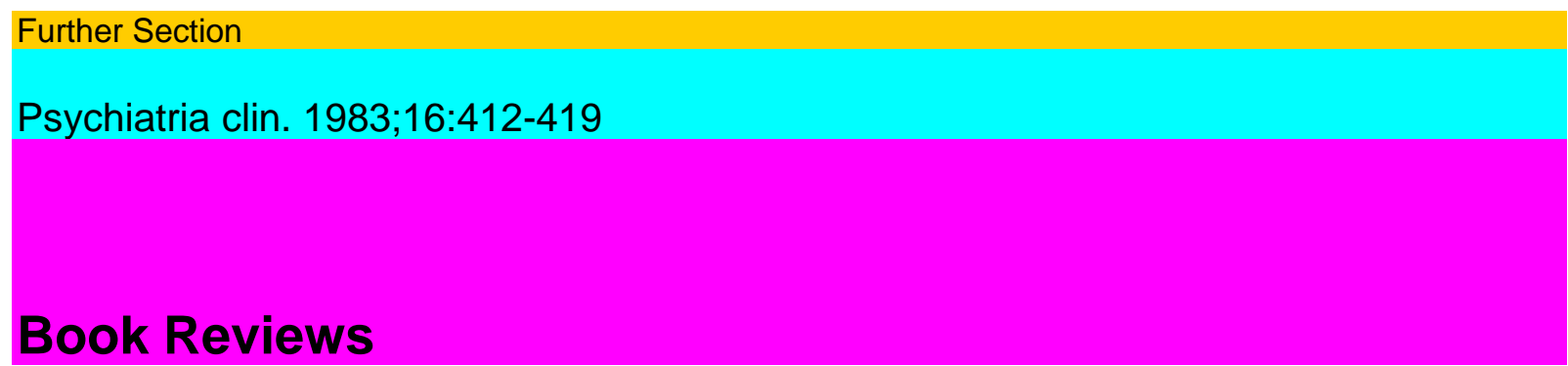

\title{
G. Göllnüz
}

Neuropsychiatrie des Kindes- und Jugendalters; 4. Aufl.

Fischer Stuttgart, 1981. 440 pp.; DM 59.-

ISBN 3-437-00340-2

In überarbeiteter und um die Kapitel «Kopfschmerzsyndrom» und «Traumatische Hirn- und Rückenmarksschäden» erweiterter Auflage ist das Lehrbuch der Neuropsychiatrie des Kindesund Jugendalters von G. Göllnitz unter Mitarbeit der Professoren Klein-peter, Olischer und Rössler neu erschienen. Wenn auch eingangs betont wird, dass eine komplette Darstellung aller Untersuchungsmethoden sowie aller neurologischer und psychiatrischer Syndrome den Rahmen eines Lehrbuches sprengen würde, wird der fur die Praxis wichtige Bereich neuropsychiatrischer Störungen behutsam ausgebreitet. Hervorzu-heben sind die grosse Überschaubarkeit und die deshalb rasche Informationsmöglichkeit, welche dieses Buch gewährt, der Einschluss entwicklungspsychologischer Erkenntnisse in die Gesamtsicht des kranken Kindes, so dass sich daraus eine bessere Zugangsmöglichkeit zum Kranken ergibt. Ausserdem wird hinter den einzelnen psychischen und neurologi-schen Störungen jeweils das Gesamtbild des kranken jungen Menschen herausgearbeitet. Jede Etikettierung und somit das Aufkeimen von Vorurteilen werden vermieden, wie sich bei der Besprechnung des Psychopathiebegriffes besonders zeigt. Aus der Einstellung der einzelnen Autoren wird spürbar, dass dieses Lehrbuch aus einer reichen Erfahrung heraus geschrieben worden ist; es will praxisnah informieren und steht über modischen Konzes-sionen. Dies kommt z.B. in den kritischen Einstellungen zu den abnormen psychogenen Entwicklungen, wie der Autor die Neurosen benennt, deutlich zum Ausdruck. Wenn die therapeutischen Möglichkeiten jeweils auch nur in Umrissen angefügt worden sind und man einzelne Hinweise wie auf den REM-Schlaf vermissen muss, kann man dieses Lehrbuch als eine integrierende Darstellung der Neuropsychiatrie des Kindes- und Jugendalters heute jedem ans Herz legen, der sich aus ärztlicher oder psychologischer Sicht mit heranwachsenden jungen Menschen beschäftigt.

H.G. Zapotoczky, Wien

J. Mendels, J.D. Amsterdam

The Psychobiology of Affective Disorders

Pfizer Symposium on Depression, Miami, Fla., 1980

Karger, Basel 1980

220 pp., 27 fig., 24 tab.

SFr. 39.-/DM 47.-/US\$ 23.50

ISBN 3-8055-1400-X

This volume presents a review of current knowledge concerned with psychobiologic findings in depression. A total of 14 contributions, part of a symposium held in Florida, cover various aspects of research on neurotransmitters, receptors, hormones and genetic factors, some of which may possibly be involved in the pathogenesis of this heterogenous

Book Reviews 


\section{3}

illness called depression. Outstanding investigators, mainly American, discuss the recent findings in a spirit of optimism and with the confidence that the identification of biologic factors - in the near future - may provide a more rational approach to diagnosis and treatment of depression.

The book starts out with a paper on epidemiological aspects; possible reasons are discussed for the apparent increase in the incidence of depression. In the following contributions, findings of clinical and biochemical studies in unipolar and biopolar depressives are presented. A review on genetic factors concludes the current evidence from a clinical perspective: None of the presently identified 'markers' of genetic vulnerability to affective disorders can be safely used in the individual to diagnose depression in the absence of adequate psychopathological symptoms. Biochemical aspects are presented by various papers: The functional activity of the noradrenergic, serotonergic and cholinergic system was studied and - hypothetically - related to etiological mechanisms. Neuroendocrine aspects of depression are discussed by a paper dealing with the dexamethasone suppression test; it is believed that this test of the functional activity of the hypothalamus-pituitary-adrenal axis may aid - if positive - in the diagnosis of endogenous depression.

Pharmacokinetic and pharmacodynamic aspects of tricyclic antidepressants (mainly doxepin) are also presented; in particular, the effects on adrenergic receptors gained special attention. Two papers deal with disturbances in sleep and circadian rhythm; the possible involvement of these factors in the pathogenesis of depression is discussed. The spectrum of contributions ends with papers discussing the applicability of psychobiological findings in clinical practice; new vistas are opened on future research strategies and data computation.

This well edited collection of papers, of theoretical and clinical value alike, provides the reader with an up-to-date summary of current psychobiologic approaches to the study of affective disorders.

Fields of Interest: Psychiatry, Clinical Chemistry, Neuroendocrinology, Pharmacology, Psychology, Psychosomatics.

F. Resch, Wien

M. Linden, M. Hautzinger Psychotherapie Manual

Sammlung psychotherapeutischer Techniken und Einzelverfahren

Springer, Berlin 1981

320 pp.; DM48.-/USS 20.50

ISBN 3-540-10683-9

Mit diesem Werk wollen die Herausgeber der Tendenz entgegenwirken, psychothera-peutisches Handeln - wie es noch immer häufiger geschieht - mit dem Schleier des Geheimnisvollen zu umgeben und zur Kunst hochzustilisieren, so dass eine detaillierte und konkrete

Durchführungsbeschreibung nicht mehr erkennbar ist. Deshalb werden in diesem - 320 Seiten umfassenden - Buch insgesamt 62 psychotherapeutische Einzelverfahren, alphabetisch geordnet, solcherart beschrieben, dass es den Charakter eines handlungs-orientierten Nachschlagewerkes erhält. Jede Technik wird zunächst allgemein dargestellt, worauf nach Hinweisen zur Indikation und Kontraindikation genaue Erläuterungen zur

Book Reviews

414 
praktischen Durchführung folgen. Die Anführung von Erfolgskriterien sowie Angaben zum Grad der empirischen Absicherung und zur weiterführenden wissenschaftlichen Literatur ergänzen das Bündel der Perspektiven, aus dem jede Technik betrachtet und beschrieben wird.

Das Selbstverständnis psychotherapeutischen klinischen Handelns, das sich in die-sem Werk niederschlägt, ist weiters geprägt von dem Bemühen um Überwindung der Divergenzen zwischen den verschiedenen psychotherapeutischen Schulen. Dementspre-chend finden sich Techniken aus den verschiedensten psychotherapeutischen Schulrich-tungen wie der Verhaltenstherapie, der Psychoanalyse, dem Autogenen Training, dem Bio-Feedback und der klientenzentrierten Spieltherapie in diesem Band versammelt.

Ein anderer wichtiger Aspekt dieses Manuals verdient noch hervorgehoben zu werden. Es richtet sich nämlich nicht nur an Psychotherapeuten, Psychotherapiestudenten und interessierte Laien, sondern auch an jene sogenannten «Mediatoren», die im Rahmen von Psychotherapien KoTherapeutenfunktionen wahrnehmen, das sind z.B. Krankenpflege-personen, aktivierende Therapeuten, Familienangehörige und andere mehr. Das Manual kann die Einweisung dieser Mitarbeiter durch den verantwortlichen Psychotherapeuten erleichtem, vor allem deshalb, weil die Handlungsanweisungen bei der Darstellung der einzelnen Beiträge im Vordergrund stehen, während der theoretische Hintergrund auf das unerlässliche Mindestmass reduziert ist. Insgesamt somit ein Werk, das vielleicht wesent-lich dazu beitragen könnte, die Verständigungsschwierigkeiten zwischen Psychotherapeuten verschiedenster Herkunft zu überwinden.

K. Purzner

J.M. Ferguson, C. Ban Taylor

The Comprehensive Handbook of Behavioral Medicine

vol. 2: Syndrome and Special Areas

MTP Press, Lancaster 1981. 289 pp.; E 15.95

ISBN 0-85200-541-5

Der 2. Band der Publikationsserie «Behavioral Medicine», die in dieser Zeitschrift bereits angezeigt wurde, umfasst vier Bereiche: psychologische Aspekte bei der Interpretation und Behandlung des Bronchialasthmas, Störungen des gastrointestinalen Systems ein-schliesslich Essstörungen, eine Einführung in das Schmerzsyndrom (chronischer Schmerz, Migräne, Spannungskopfschmerz und Möglichkeiten von verhaltensmedizinischen Inter-ventionen von Schmerzzuständen in der Zahnheilkunde) und nicht zuletzt Hautstörungen psychosomatischer Genese wie Dermatosen und Verhaltensstörungen, welche - wie Nägel-beissen - ebenfalls die Haut betreffen. Also wiederum vier Themata, die in der Praxis eine grosse Rolle spielen und denen gegenüber das therapeutische Bemühen bisher im allgemei-nen recht erfolglos war. Es ist nicht immer möglich, therapeutische Grundkonzepte anzubieten: Versucht wird dies im Bereich Ubergewichtigkeit mit der Darstellung des Stanford Adolescent Obesity Project (Coates und Thoresen), bei kachektischen Patienten mit Hilfe eines The-rapiekontraktes (Ferguson), mit einem differenzierten Behandlungsprogramm bei verschiedenen Enkopretikertypen (Doleys) sowie mit Kontraktprogrammen bei verschiedenen Schmerzzuständen. Durch diese Darstellungen werden neue Wege aufgezeigt, die von

Book Reviews

415

eingefahrenen und oft auch erfolglosen Behandlungsschablonen wegführen - oder zumin-destens zu neuen Überlegungen anregen. 
Die meisten Beiträge dieses Bandes zielen zunächst auf einen Literaturüberblick der bisherigen psychotherapeutischen, psychopharmakologischen und verhaltensmedizini-schen Behandlungsversuche ab. Es wird durchgehend das Fehlen von kontrollierten Stu-dien beklagt; die meisten Erkenntnisse beruhen auf Einzelfalldarstellungen. Gefordert werden ein psychotherapeutisches Eingreifen und mögliches Identifizieren von potentiellen Risikogruppen (z.B. von potentiellen chronischen Schmerzpatienten), bevor chirurgische Massnahmen ergriffen werden oder sich eine chemische Abhängigkeit von verschiedenen Substanzen entwickeln kann. Mit einigen Vorurteilen wird aufgeräumt: Die Bedeutung psychologischer Faktoren bei der Entstehung des Asthmas (Alexander und Solanch), die Annahme eines konditio-nierten Bronchospasmus werden in Frage gestellt und grösserer Wert auf die psychologi-schen Konsequenzen der Anfälle selbst gelegt. Oder: Wenigstens kurzfristig sind verhaltensmodifizierende Techniken bei Übergewichtigen anderen Methoden wie Plazebo-, traditionellen Diätgruppen, auf Einsicht beruhende Therapiegruppen, Informationsgruppen und Kontrollgruppen ohne Therapie überlegen, wenngleich die Behandlungsversuche nach wie vor ungenügend und beinahe entmutigend genannt werden können.

Alles in allem: Wiederum ein Band anregender Kapitel aus medizinisch-psychologi-schen Grenzbereichen, deren Behandlungsmöglichkeiten weiterdiskutiert werden müssen. Die Referate von berufenen Fachleuten liefern den entsprechenden Zündstoff dazu.

H.G. Zapotoczky, Wien

E. Bomeman Reifungsphasen der Kindheit

Sexuelle Entwicklungspsychologie, vol. 1 Sauerländer, Aarau 1981. 263 pp. SFr. 39.80 ISBN 37941-2254-2

Der Versuch, die sexuelle Entwicklung des Menschen in präzise, nach Altersstufen gegliederte Phasen einzuteilen, ist im psychoanalytischen Schrifttum nicht neu. Bomeman sieht dazu drei Bände vor, die sich in Kindheit, Jugendzeit und Erwachsenenalter gliedern sollen und deren erster jetzt vorliegt. Wie der Autor eingangs bemerkt, versteht er seine Ausführungen als «vorbereitende Arbeit für die Errichtung» von Lehrstühlen für Sexual-pädologie. Unabhängig von der Überlegung, inwieweit Bücher überhaupt die Errichtung von Lehrstühlen fördern können oder nicht, scheint die Frage angebracht zu sein, ob die Darlegungen ein besseres Verständnis für die sexuelle Entwicklung des Menschen ermög-lichen. Und das muss leider bezweifelt werden. Schon der erste Satz stimmt nicht ganz. Bereits vor Bomeman wurde versucht, die sexuelle Entwicklung des Kindes in präzisen, nach Altersstufen gegliederten Phasen zu beschreiben. Es sei nur an Charles Baudouin erinnert oder an Françoise Marette-Dolto, zwei Autoren, die bis in die letzte Zeit neue, orientierende Gesichtspunkte der Libidoent-wicklung eingebracht haben, allerdings in Bornemans Personenregister nicht erwähnt sind. Oder was soil von Behauptungen gehalten werden wie die, dass sich die Freude der Mutter an ihrem Geschlechtsleben während der Schwangerschaft in der sexuellen Vitalität des

Book Reviews

416

Kindes niederschlägt, wenn man nichts darüber erfährt, welche Erhebungsmethoden zu dieser Feststellung geführt haben. Die Idee ist ja an und fur sich köstlich, aber triíit sie auch zu? Eine Seite später heisst es, dass die Geburt einem 15minütigen Sauerstoffentzug ent-spricht, was mit Sicherheit zum Tod des Kindes fiihren würde. Genügte dem Autor die präzisere Bezeichnung «Sauerstoffmangel» oder «Drosselung der Sauerstoffzufuhr» nicht?

Eine weitere Behauptung, die zu Unklarheit und Verwirrung fiihren kann, ist diejeni-ge, dass der Knabe «bis zur Geburt sogar organischer Teil» des Körpers der Mutter sei; diese Darstellung ist 
sicherlich falsch. Ob Knabe oder Mädchen, ein Kind ist niemals organischer Teil des mütterlichen Körpers.

Wenn das Buch der Ausbildung von Lehrern im schulischen Sexualunterricht nützen will, worauf im Vorwort hingewiesen wird, sind Missverständnisse der beschriebenen Art bedauerlich und die Frage berechtigt, ob man sich nicht lieber gleich an jene Literatur halt, die gesichertes Wissen über die Libidoentwicklung des Menschen in weniger problemati-scher Form darzustellen weiss.

H.G. Zapotoczky, Wien

Jean Piaget

Intelligence and Affectivity

Their Relationships during Child Development Annual Reviews, Palo Alto 1981. 75 pp.; US\$

9.00 ISBN 0-8243-2901-5

Das vorliegende Buch beruht auf Vorlesungen Jean Piagets an der Sorbonne in den Jahren 1953 und 1954; der französische Originaltext wurde 1957 im «Bulletin de Psycho-logie» veröffentlicht. Es ist der Entwicklung der Affektivität und ihrer Beziehung zu den Entwicklungsstadien der Intelligenz gewidmet.

Im Vorwort (Ph. A. Cowan) werden die zentralen Gedanken zusammengefasst: Der Affekt funktioniert als Energielieferant der intellektuellen Prozesse und wird durch die Disequilibration von Assimilation und Akkommodation aktiviert. Somit sind Kognition und Affekt als zwei Seiten einer Medaille zu sehen. Die Entwicklung von Kognition und Emotion zeigt parallele Stufenabfolgen von der Geburt bis zur Adoleszenz.

Bevor wir einzelne inhaltliche Aspekte hervorheben, sei uns eine verkürzte Zusam-menfassung des Gesamteindrucks gestattet: Wenn auch bei dieser Themenstellung das systematische Konzept Piagetschen Denkens besticht, so tritt doch die diffizile, analysie-rende Darstellung zugunsten globaler Analogien und Vergleiche in den Hintergrund. Dies beeinträchtigt in keiner Weise die Faszination der Darstellung und die ungemein anre-gende Entwicklung eigener Positionen in Abgrenzung von anderen Auffassungen (Freud, Adler, Claparede).

Piaget schreibt in der Einleitung, dass kognitive und affektive Faktoren im konkreten Verhalten des Individuums wohl untrennbar sind, es sich aber dennoch um unterscheid-bare Funktionen handelt. Er beantwortet die Frage der Relation der beiden Funktionen dahingehend, dass die Affektivität die Rolle einer Energiequelle spielt, von der die Funk-tion, aber nicht die Struktur der Intelligenz abhängig ist. Somit stammt die Energie des Verhaltens aus der Affektivität. die Strukturen jedoch von den kognitiven Funktionen. Die Book Reviews

417

Annahme, dass aîiektive Phänomene angeboren sind, bezeichnet Piaget als ein romanti-sches Vorurteil.

Parallel zum Stufenmodell der kognitiven Entwicklung nimmt Piaget auch affektive Entwicklungsstufen an, wobei den beiden Hauptkategorien, der sensomotorischen Intelli-genz und der verbalen Intelligenz, einerseits die intraindividuellen Gefühle, andererseits die interpersonalen Gefühle zugeordnet und in jeweils drei Untergruppen gegliedert werden. In der Analyse der ersten Entwicklungsstufe betont Piaget die Differenzierung des englischen Begriffs «instinct», der einerseits die Koordination von Reflexen zur Befriedi-gung eines Bedürfnisses meint, andererseits den deutschen Begriff «Trieb» umfasst und somit das Bedürfnis selbst und gleichzeitig das energetische Element eines Instinkts meint. Piaget bezieht sich nun auf einen Instinktbegriff im engeren Sinne, fur den er folgende Kriterien fordert: den 
spezifischen Trieb, eine gut defmierte Verhaltensweise in Form von sensomotorischen Strukturen sowie differenzierte Organe. Unter dieser Prämisse subsu-miert er unserem Instinktbegriff das Begriffspaar Nahrung/Hunger sowie Sexualität, nicht jedoch die komplexen Dimensionen Selbsterhaltung, Verteidigung, Neugier, Spielinstinkt, Sozialinstinkt usw. Im Kapitel über die erworbenen Gefühle postuliert Piaget eine Komplexitätsstufen-leiter, die von der Empfindung über die Wahrnehmung zum Gefühlszustand führt und keine grundsätzlichen Differenzen beinhaltet.

Trotz der bereits einleitend erwähnten Unterscheidung zwischen affektiven und kognitiven Aspekten des Verhaltens lehnt Piaget eine dualistische Position ab und nimmt vielmehr eine konstante und dialektische Interaktion zwischen Affektivität und Intelligenz an. Die Periode der erworbenen Empfindungen entspricht der der primären und sekundä-ren Zirkulärreaktionen. Der Inhalt des dritten Stadiums ist die Differenzierung und Dezentrierung der Affek-te, sein Kennzeichen ist die Entstehung eines Wertsystems, das nicht der unmittelbaren Handlungsökonomie, sondern den Zielsetzungen entspricht und das die Energie bestimmt, die in eine Aktion investiert wird. Die Entstehung dieses Wertsystems wird anhand der Theoriekonzepte von Kurt Lewin und Sigmund Freud diskutiert. Insbesondere die Diskus-sion Freudscher Positionen ist von höchstem Interesse: Die mangelnde Erinnerung an Erlebnisinhalte aus dem 1. Lebensjahr ist nicht durch Verdrängung, sondern durch das Fehlen eines evokativen Gedächtnisses, das auf Symbolfunktionen beruht, erklärbar. Das Konzept des primären Narzissmus übersieht die Tatsache, dass zu diesem Zeitpunkt das Selbst noch nicht formiert ist, somit der «Narzissus» fehlt. Zum Thema «Objektwahl» formuliert Piaget eine Alternativkonzeption zur Libidoverschiebung; das «Objekt» ist ein polysensorischer Komplex, der unabhängig von der unmittelbaren Wahrnehmung exi-stiert; somit ist anstelle einer affektiven Objektwahl eine affektive und kognitive Ausarbei-tung des Objektes («Objektkonstruktion») anzunehmen.

In der nächsten Stufe entwickeln sich parallel zur Sprache und zur Repräsentation die interpersonalen und sozialen Gefühle. Voraussetzung ist die Objektrepräsentation und die Bewahrung der Gefühle auch ohne Präsenz der auslösenden Objekte. Unter dem Begriff der normativen Gefühle werden die Adlerschen Konzepte des Minderwertigkeitsgefühls und des Machtstrebens besprochen, unter dem Begriff der Moralgefühle folgt eine Diskus-sion des Freudschen Konzepts des Unbewussten; hier geht Piaget davon aus, dass nicht Gefühle gespeichert werden, sondern Schemata der Interaktion mit anderen Personen, die bei bestimmten Gelegenheiten rekonstruiert werden. Es folgt eine Kurzdarstellung der Moralentwicklung. Book Reviews

418

Zwischen dem 7. und 12. Lebensjahr stehen neben dem autonomen Moralempfinden die Bewahrung von Gefühlen sowie das Problem des Willens zur Diskussion - «Der Wille ist einfach das affektive Analogon der intellektuellen Dezentrierung.» Ab dem 11.-12. Lebensjahr erfolgen sowohl im affektiven als auch im kognitiven Bereich Transformatio-nen in Richtung der formalen Operationen.

In der Zusammenfassung setzt sich Piaget schliesslich noch mit dem Begriff der Regression auseinander, dessen Anwendung er dann fur berechtigt halt, wenn Konflikte zwischen Elementen unterschiedlicher Niveaus - z.B. einer mentalen Operation eines höheren und eines Gefühls eines niedrigeren Niveaus - bestehen. Nochmals weist er darauf hin, dass affektive Strukturen keine spezielle Art von Strukturen darstellen und das Verhalten zu Objekten ebenso wie zu Personen jeweils kognitive/strukturelle und affektive/energeti-sche Aspekte umfasst. 


\section{E. Berger, Wien \\ G. Pirooz Sholevar \\ The Handbook of Marriage and Marital Therapy \\ MTP Press, Lancaster 1981552 pp.; E23.75 ISBN 0-85200-567-9}

Dieses Handbuch stellt eine Sammlung der wichtigsten Aspekte dar, die dem Thema Paar/Partnertherapie heute abgewonnen werden können. Immerhin sind 31 amerikanische Autoren verschiedenster professioneller Provenienz an dieser Kollektion beteiligt; nicht immer sind sie Fürsprecher, häufig auch Kritiker dieser neuen, wichtigen Therapierich-tung. So erhebt zum Abschluss des ersten Kapitels, welches auf psychodynamischen Theo-rien basiert, Otto Pollak seine warnende Stimme: Er verweist darauf, dass Familienthera-peuten das industrielle Produktionsmodell zur Evaluation ihrer Arbeit verwenden. «ln human affairs the principle of indeterminacy is always at work.» Man muss sich fragen, ob in derartige Äusserungen nicht doch gewisse Vorurteile einfliessen, gerade, wenn sich im Literaturverzeichnis der psychodynamisch orientierten Autoren kein Hinweis auf die Befunde von Willi oder Staehelin findet.

Im zweiten Abschnitt werden verschiedene andere paartherapeutische Methoden geschildert. Bereits die Lektüre des Beitrags von Whitacher, Greenberg und Greenberg eröffnet hilfreiche Einsichten in verschiedene Grundhaltungen; Stanton vertritt einen systemtheoretisch orientierten Standpunkt; Magran hebt transaktionsanalytische Aspekte hervor. Verhaltenstherapeutische Techniken fasst Epstein zusammen.

Den Abschluss bilden Erörterungen spezieller Techniken und Ergebnisse. Williams und Miller stellen das offensichtliche Desinteresse klinischer und psychologischer Institu-tionen an der Paartherapie heraus. Sie fordern Längsschnittstudien, die experimentell aus-gerichtet sind und sich mit Teilaspekten befassen. Ein lesenswertes Kapitel! Ficher und Coleman räumen - man ist beinahe versucht zu sagen: wieder einmal - mit Vorurteilen betreffend sexuelle Aktivitäten z.B. in der Unterschicht auf und betonen - zum wievielten Mai? - die Machtverhältnisse hinter Paarbildungen und Sexualität. Auch Adler wird im Literaturverzeichnis nicht erwähnt! Book Reviews

419

Sholevar schildert die Folgen, welche das Zerbrechen familiärer Strukturen flir Kinder haben kann - nicht nur im Moment dieses Ereignisses, sondern auch im Hinblick auf die kommende eigene Partnerbeziehung: Mangelndes festumrissenes Familienbild, ge-schwächtes SelbstKonzept und gestörte Geschlechtsidentität sind ebenso zu beklagen wie eine höhere eigene Scheidungsrate. Interventionsstrategien und Möglichkeiten der Prevention werden angeführt, welche diese Entwicklungen zumindest abschwächen sollen. Ein Pflichtkapitel fur jeden Kindertherapeuten, Pädagogen und Elternteil! Freedman be-schreibt neurotische Probleme in der Ehe - auch hier bleibt Willi unerwähnt. Die letzten Kapitel befassen sich mit Gewalttätigkeit in der Partnerschaft (McLeer), auch bei uns ein offenbar immer öñer geäussertes Problem, und ausserdem mit der Ehe zweier berufstätiger Partner und deren Einfluss auf die Entwicklung der Kinder (Fishkin und Fishkin) - eine aktuelle Schilderung der Gefáhrdungen und deren Vermeidung.

Z. Zusammenfassend ist über ein Handbuch nordamerikanischen Zuschnitts zu bench-ten das wegen einiger auch fur uns aktueller Probleme lesenswert ist im übrigen eine Selbstdarstellung einer modernen Therapieform wiedergibt zu der auch europäische The-rapeuten Wichtiges und fur uns Gültiges geäussert haben; sie bleiben unerwähnt.

H.G Zapotoczky 
Wien 